\title{
Claude Langlois, Le continent théologique. Explorations historiques
}

Rennes, Presses universitaires de Rennes, coll. « Histoire », 2016, 358 p., préface de Guillaume Cuchet et Denis Pelletier.

\section{Sylvain Milbach}

\section{(2) OpenEdition}

Journals

Édition électronique

URL : http://journals.openedition.org/assr/28289

DOI : $10.4000 /$ assr.28289

ISSN : $1777-5825$

Éditeur

Éditions de l'EHESS

Édition imprimée

Date de publication : 31 décembre 2016

Pagination : 346

ISSN : 0335-5985

\section{Référence électronique}

Sylvain Milbach, "Claude Langlois, Le continent théologique. Explorations historiques », Archives de sciences sociales des religions [En ligne], 176 | octobre-décembre 2016, mis en ligne le 20 juillet 2017, consulté le 21 décembre 2020. URL : http://journals.openedition.org/assr/28289; DOI : https:// doi.org/10.4000/assr.28289

Ce document a été généré automatiquement le 21 décembre 2020.

(C) Archives de sciences sociales des religions 


\section{Claude Langlois, Le continent théologique. Explorations historiques}

Rennes, Presses universitaires de Rennes, coll. « Histoire », 2016, 358 p., préface de Guillaume Cuchet et Denis Pelletier.

\section{Sylvain Milbach}

\section{RÉFÉRENCE}

Claude Langlois, Le continent théologique. Explorations historiques, Rennes, Presses universitaires de Rennes, coll. « Histoire », 2016, 358 p., préface de Guillaume Cuchet et Denis Pelletier.

1 Claude Langlois a enseigné à l'université de Créteil (1972-1984), puis à celle de Rouen (1985-1993), avant de devenir directeur d'études à l'École Pratique des Hautes Études (1993-2005), dont il a dirigé la Section des sciences religieuses, où il a fondé avec Régis Debray l'Institut européen en sciences des religions (2002). Ce parcours académique est rappelé en préface par Guillaume Cuchet et Denis Pelletier, qui insistent spécialement sur le dynamisme de l'historiographie du christianisme dans l'université française dans la génération d'historiens en activité à partir des années 1970. L'auteur revient luimême sur ce parcours en une brève introduction qui prolonge l'analyse d'un parcours historiographique dont le Continent théologique propose quelques applications.

2 L'ouvrage rassemble vingt-trois articles publiés entre 1973 et 2014, choisis dans une bibliographie d'environ 300 titres qui complète l'ouvrage. Ces articles sont accompagnés de notes ou d'introductions qui précisent la démarche et le contexte qui ont présidé à leur rédaction. Ces précisions affinent les deux premiers chapitres "d'ego-histoire », et sont utiles pour comprendre un cheminement original, car le lecteur peut se sentir décontenancé, et admiratif, devant la variété, et souvent la singularité, des sujets abordés. Le recueil ouvre par ailleurs un vaste éventail 
chronologique, de la fin du XVIII ${ }^{e}$ siècle au XXI ${ }^{e}$ siècle. Le "temps présent" permet d'éclairer la démarche de l'historien, mais il met aussi l'accent sur la durée des objets "sociétaux ». En effet, tous les sujets traités profitent d'une mise en profondeur historique, en amont comme en aval. Les articles sont regroupés en sept thématiques où dominent les deux centres d'intérêt de l'auteur: la théologie morale et l'écriture mystique.

3 Dans ce vaste éventail, se distingue d'abord le premier $\mathrm{xIX}^{\mathrm{e}}$ siècle, spécialement en matière de théologie morale, qui fut la période de prédilection initiale de Claude Langlois. L'analyse porte surtout sur les conditions de la production du discours théologique qui s'inscrit dans une double conjoncture: les transformations sociologiques et l'évolution des comportements dans la société post-révolutionnaire; les procédures de construction et de validation des formulations théologiques, dans une sorte de période de transition entre le xviII ${ }^{e}$ siècle, où Rome juge en dernière instance des débats, et le dernier tiers du $\mathrm{xIx}^{\mathrm{e}}$, puis le $\mathrm{xx}^{\mathrm{e}}$ siècle, où Rome juge en première instance. Cette double conjoncture laisse un espace de liberté à une théologie morale innovante et à l'initiative de théologiens (Mgr Bouvier, Mgr Gousset) qui prennent la parole de leur propre initiative. On retrouve également au fil de ce volume ce qui est un des axes majeurs de la recherche de Claude Langlois, ces « catholicismes au féminin » dont il fut un pionnier. Il est impossible de rendre compte ici par le menu de la densité des articles réunis, souvent des «études de cas» qui fourmillent de notations suggestives. On préférera ici rendre compte, même succinctement, de quelques points de méthode qui donnent à cette réunion d'articles une vraie cohérence. Le «continent théologique» est une terre où les historiens de la période contemporaine ne sont pas nombreux à s'aventurer, et moins nombreux encore à la prendre au sérieux, sinon, peut-être, pour évoquer les crispations qui, au moins du syllabus au concile Vatican II, mettent l'Église en délicatesse avec le mouvement des idées de la société contemporaine. Sans contourner ces lieux de crispation (le Syllabus, l'infaillibilité pontificale, l'affaire Loisy, la sexualité, le statut des femmes), Claude Langlois propose une approche plus apaisée en s'efforçant de dégager les logiques propres à l'Église, en réelle dialectique leur temps.

5 Cette démarche contribue à historiciser la production théologique, c'est-à-dire à la concevoir comme une réponse pragmatique à des défis nouveaux. Cette dialectique est d'autant plus difficile à saisir que la formulation théologique a, par essence, tendance à expulser l'explication contingente au profit de la référence à la tradition et à gommer de ce fait les aspérités de l'histoire. Pourtant, la tension entre la norme, issue du noyau intangible de la Révélation, et ses applications dans des sociétés humaines - la France pour l'essentiel dans ce volume -, dont les valeurs deviennent plus fluides et entrent en concurrence, est pour l'historien le lieu pour cerner la réactivité de l'Église aux phénomènes sociaux, et ses hésitations. Il lui revient donc de rétablir les conditions de la production théologique et dogmatique dans ses causes, sa mécanique et ses conséquences. "Ce qui est en cause c'est tout à la fois la capacité du discours clérical, dans sa formalisation théologique, à avoir prise sur le réel [...] et tout autant sa possibilité de donner sens au changement des pratiques sociales ", écrit Claude Langlois (p. 119) à propos d'une recherche débutée sur l'Église et le prêt à intérêt : cette formule me semble résumer l'ensemble de la démarche de l'historien. Finalement, c'est dans l'article le plus ancien, celui de 1973, significativement le seul pour les décennies 1970 et 1980 , sur l'infaillibilité pontificale, que l'auteur formule son projet avec plus de 
netteté en exposant sa «conviction que la démarche du théologien - à tout le moins de certains d'entre eux - impliquait une sous-estimation de l'histoire » (p. 51).

6 C'est aussi par l'inscription dans le temps long qu'apparaît au mieux l'effet des conjonctures : c'est le cas dans l'étude de l'effritement de la thèse du " petit nombre des élus », critiqué par Bergier dès la fin du XVIII ${ }^{\mathrm{e}}$, mais qui s'impose fort lentement devant les «cas de conscience» posés par la modernité (où joue spécialement le statut de l'enfant in utero - avortement et fécondation in vitro - qui interroge à nouveaux frais le salut des petits êtres). Le même type d'aggiornamento, en réalité un lent et presque insensible déplacement du discours, est mis en valeur dans la très belle contribution sur "Catholicisme, douleur et dolorisme », où est analysé avec beaucoup de nuances le discours de l'Église sur le soulagement de la douleur jusqu'à Pie XII.

7 Dans les fondations de l'écriture de l'histoire par Langlois réside l'intérêt porté aux causes démographiques, qui explique précisément cette attention au temps long et la contextualisation des constructions théologiques. Son histoire religieuse et culturelle est d'abord une histoire sociale au sens fort du terme, qui ne se limite pas aux représentations induites par les changements comportementaux, mais qui en dégage les causes sociologiques et les conséquences sur le positionnement de l'Église. L'étude du discours sur la sexualité conjugale, entre le XVIII siècle et Vatican II, met à l'œuvre cette analyse et montre comment le discours théologique s'adapte lentement, et difficilement, à un fait de société massif - la limitation des naissances - produisant un double corpus de référence: une mémoire théologique stricto sensu (les usages des références scripturaires - le "crime d'Onan ») et une mémoire institutionnelle (la sédimentation des avis confidentiels de la Sacrée Pénitencerie). Au $\mathrm{xx}^{\mathrm{e}}$ siècle, la seconde mémoire tend à recouvrir la première, et, là encore, insensiblement, le discours ecclésial se déplace en maintenant le rapport à la tradition (les «croyances intouchables»). La volonté de comprendre "de l'intérieur» de l'institution, en s'attachant à ses logiques propres, les positions de l'Église est un des points forts de la démarche.

Un autre axe d'analyse, dans l'ordre méthodologique, réside dans l'attention portée aux textes : « En revenir au texte », " au dire », dans le cas de sainte Thérèse de Lisieux, ou « lire tout simplement ", pour reprendre la formulation utilisée dans l'article consacré au Syllabus, exemplaire selon moi de la démarche. Il ne s'agit pas en effet de reprendre ce texte célèbre par sa réception, qui fait écran à l'intelligence du texte, ni même seulement par les modalités de son élaboration, mais de le concevoir comme un énoncé de positions où sont tranchés aussi, et peut-être d'abord, des débats internes à l'Église. L'analyse du texte dégagée de sa réception polémique rend compte de ses spécificités : original dans sa composition au regard des textes antécédents de condamnation doctrinale ; original parce que s'y dessinent les conceptions de la catholicité autant que des condamnations des "erreurs modernes». Ce retour au texte, démarche au fondement des travaux «thérésiens » de Langlois, tient à la conviction qu'il existe une "étrangeté textuelle", particulièrement évidente en matière de théologie, tenant d'abord aux modes d'élaboration de ces textes, dont doit d'abord procéder le travail de l'historien.

9 On peut, pour finir, reprendre l'avis des introducteurs du volume: le Continent théologique témoigne pour le parcours et la méthode d'un grand historien, qui sait restituer les logiques des institutions dans leurs liens avec les phénomènes sociaux, et en réduire l'étrangeté. Celle-ci est particulièrement vive dans le domaine théologique 
qui relève souvent d'une « culture » de la longue durée qui échappe de plus en plus à la société contemporaine, médiatique, "présentiste ", et réactive à outrance. Les articles réunis permettent souvent d'aborder sous un autre front le dialogue difficile entre des conceptions intemporelles de l'humanité et les aspirations qui la travaillent.

10 Langlois a largement frayé son chemin indépendamment des modes - et l'ouvrage n'en donne qu'un aperçu-, des cadres disciplinaires académiques et des injonctions de l'institution universitaire pour produire une œuvre originale. L'auteur l'exprimait en 2007 dans le court essai d'ego-histoire proposé en tête de volume: «la recherche vraiment innovante est sans doute celle qui n'est pas programmée, celle qui d'abord s'impose, comme malgré elle, à celui qui se laisse entraîner par le mouvement même de cette inlassable curiosité qui est le ressort de toute vie intellectuelle. Et de ce fait, elle est, dès l'abord, inaudible, incompréhensible. Et c'est là que l'institution prend tout son sens ». 